\title{
Erratum to: Nonplanar solitons in a warm electronegative plasma with electron nonextensivity effects
}

\author{
S. Ali Shan ${ }^{1,2,3}$ • Aman-ur Rehman ${ }^{3}$
}

Published online: 27 November 2015

(C) Springer Science+Business Media Dordrecht 2015

Erratum to: Astrophys. Space Sci. (2014) 352:593-604

DOI 10.1007/s10509-014-1968-x

In our original paper (Shan and Rehman 2014), we studied nonplanar solitons in a warm electronegative plasma with $q$ distributed nonextensive electrons. In our discussion about the relation (13) of our paper for the case of nonplanar geometry,

$$
\begin{aligned}
k(k & \left.-\frac{i p}{r}\right)+\delta \mu \frac{(q+1)}{2} \\
= & \frac{\delta \nu k\left(k-\frac{i p}{r}\right)}{\omega^{2}-3 \theta_{n} k^{2}+3 i k \theta_{n}\left(\frac{p}{r}\right)} \\
& +\frac{\delta \alpha k\left(k-\frac{i p}{r}\right)}{\omega^{2}-3 \alpha k^{2} \theta_{i}+3 i \alpha k \theta_{i}\left(\frac{p}{r}\right)},
\end{aligned}
$$

we have mentioned that the dispersion relation (1) associated with the IA waves in electronegative plasma is strongly affected by the radial dependence $(r)$ and the geometrical ef- fects $(p)$. It is clear from Eq. (1) that linear harmonic wave method only works when the irritating imaginary terms $(i p / r)$ disappear. However, there are two possibilities by which one can eliminate the imaginary terms, either one should choose $p=0$, which reverts to planar waves strictly, or else one should be allowed to have large values of $r$. In the second case, the model applies only to phenomena far from the axis of cylindrical symmetry or from the origin of (which is conventionally at $r=0$ ).

We note that these findings were first reported by Verheest and Hellberg (2014) and we consulted their research paper. We apologize to the authors of Verheest and Hellberg (2014) that we omitted their paper from our bibliography.

\section{References}

Shan, S.A., Rehman, A.: Astrophys. Space Sci. 352, 593-604 (2014) Verheest, F., Hellberg, M.: Phys. Plasmas 21, 022307 (2014)
The online version of the original article can be found under doi:10.1007/s 10509-014-1968-x.

$凶$ S.A. Shan

shaukatshan@gmail.com

1 Theoretical Physics Division, PINSTECH, P.O. Nilore, Islamabad 44000, Pakistan

2 National Centre for Physics at Quaid-e-Azam University Campus, Shahdra Valley Road, Islamabad 44000, Pakistan

3 Department of Physics and Applied Mathematics (DPAM), PIEAS, P.O. Nilore, Islamabad 44000, Pakistan 